\title{
HEREDITY OF WHITTAKER MODELS ON THE METAPLECTIC GROUP
}

\author{
WILLIAM D. BANKS
}

\begin{abstract}
In this paper, Rodier's theorem on the heredity of Whittaker models is generalized to non-algebraic setting of the $n$-fold metaplectic cover of the general linear group $G L_{r}(\mathbb{F})$, where $\mathbb{F}$ is nonarchimedean local field containing the $n$-th roots of unity.
\end{abstract}

\section{Introduction.}

Let $\mathbb{F}$ be a nonarchimedean local field, let $G$ be the general linear group $G L_{r}(\mathbb{F})$ for some positive integer $r$, and let $P$ be a standard parabolic subgroup of $G$ with Levi component $M$. Given an admissible representation $\pi_{M}$ of $M$, extend $\pi_{M}$ to a representation $\pi_{P}$ of $P$ by letting the unipotent radical of $P$ act trivially, and let $\pi_{G}$ be the normalized full-induced representation $\operatorname{Ind}\left(P, G ; \pi_{P}\right)$. Then by a well-known result of $\mathrm{F}$. Rodier, there exists a correspondence between the Whittaker models of the induced representation $\pi_{G}$ and the Whittaker models of the inducing representation $\pi_{M}$ (cf. Theorem 2 of $[4])$.

In this paper, Rodier's theorem on the "heredity" of Whittaker models is extended to the non-algebraic setting of the $n$-fold metaplectic cover $\widetilde{G}$ of $G$, where $n$ is a positive integer such that $\mathbb{F}$ contains all of the $n$-th roots of unity. The main result is stated as a theorem in $\S 2$. In order to illustrate the situation, consider the example of a representation of $\widetilde{G}$ induced from the metaplectic preimage $\widetilde{B}$ of the standard Borel subgroup $B$ of $G$. Since the Levi component $T$ of $B$ is a (maximal) torus in $G$, its metaplectic preimage $\widetilde{T}$ is a Heisenberg group. Consequently, the dimension of any irreducible representation $\pi_{T}$ of $\widetilde{T}$ is equal to the index $\left[\widetilde{T}: \widetilde{T}_{*}\right]$, where $\widetilde{T}_{*}$ is an arbitrary maximal abelian subgroup of $\widetilde{T}$. In this example, every linear functional on the space of $\pi_{T}$ is a Whittaker functional, hence the inducing representation $\pi_{T}$ has precisely $\left[\widetilde{T}: \widetilde{T}_{*}\right]$ distinct Whittaker models. Now extend $\pi_{T}$ to a representation $\pi_{B}$ of $\widetilde{B}$ (see $\S 2$ below), and let $\pi_{G}$ be the normalized, fullinduced representation $\operatorname{Ind}\left(\widetilde{B}, \widetilde{G} ; \pi_{B}\right)$ of $\widetilde{G}$. By Lemma I.3.2 of [3], it follows that $\pi_{G}$ also has $\left[\widetilde{T}: \widetilde{T}_{*}\right]$ distinct Whittaker models, thus Rodier's theorem evidently extends to this example. 
While the main techniques of proof employed in this paper are contained in [4], it is unclear a priori that those techniques carry over to the metaplectic group. Here the situation is clarified by a close examination of various aspects of Rodier's proof.

The results of this paper will be relevant to the generalization of F. Shahidi's theory of local coefficients (cf. [5]) to the metaplectic setting, to the construction of certain non-principal theta functions (cf. [3]), and to the eventual classification of metaplectic representations.

The author would like to thank J. Cogdell for some valuable discussions on the theory of distributions, and the Oklahoma State University for providing support during the preparation of this paper.

\section{The Metaplectic Group, Whittaker Models, and Heredity.}

Let $n$ and $r$ be fixed positive integers. Let $\mathbb{F}$ be a nonarchimedean local field, and let $\mu_{n}$ denote the group of $n$-th roots of unity in $\mathbb{F}$. We will assume that $\mu_{n}$ has cardinality $n$.

Let $\widetilde{G}$ denote the $n$-fold metaplectic cover of $G:=G L_{r}(\mathbb{F})$ (cf. $\S 0 . I$ of [3]). As a set, $\widetilde{G}=G \times \mu_{n}$, with multiplication defined by:

$$
(g, \zeta) \cdot\left(g^{\prime}, \zeta^{\prime}\right)=\left(g g^{\prime}, \zeta \zeta^{\prime} \sigma\left(g, g^{\prime}\right)\right), \quad \forall g, g^{\prime} \in G, \zeta, \zeta^{\prime} \in \mu_{n}
$$

Here $\sigma: G \times G \rightarrow \mu_{n}$ is the Matsumoto 2 -cocycle in $\mathrm{Z}^{2}\left(G ; \mu_{n}\right)$. Let $\mathbf{s}: G \rightarrow \widetilde{G}$ be the preferred section $g \mapsto(g, 1)$, and let $\mathbf{p}: \widetilde{G} \rightarrow G$ be the canonical projection $(g, \zeta) \mapsto g$.

Let $N$ be the unipotent radical of the standard Borel subgroup $B$ of $G$, and let $N^{\mathbf{s}}:=\mathbf{s}(N)$. Since $\left.\sigma\right|_{N \times N}=1, \mathbf{s}: N \rightarrow N^{\mathbf{s}}$ is a group isomorphism. Once and for all, let $\psi$ be a fixed principal character of $N$ (cf. $\S 2$ of [4]). Then for any positive simple root $\alpha$ of $G$, the restriction of $\psi$ to the unipotent root group $N_{\alpha}$ is nontrivial. Let $\psi^{*}$ denote the corresponding character $\psi \circ \mathbf{p}=\psi \circ \mathbf{s}^{-1}$ of $N^{\mathbf{s}}$, and let $\bar{\psi}^{*}$ be the character obtained from $\psi^{*}$ by complex conjugation.

Let $W$ be the Weyl group of permutation matrices in $G$, and let $W^{\mathbf{s}}:=$ $\mathbf{s}(W)$. If the $n$-th order Hilbert symbol $(\cdot, \cdot)_{\mathbb{F}}: \mathbb{F}^{\times} \times \mathbb{F}^{\times} \rightarrow \mu_{n}$ satisfies the relation $(-1,-1)_{\mathbb{F}}=1$, then $\mathbf{s}: W \rightarrow W^{\mathbf{s}}$ is a group isomorphism, but we will proceed without this assumption.

Let $M \subseteq G$ be the Levi component of an arbitrary standard parabolic subgroup $P$ of $G$. Then $P=M U$, and $M \cap U=\{e\}$, where $U \subseteq N$ is the unipotent radical of $P$, and $e$ is the identity of $G$. Let $\widetilde{P}, \widetilde{M}$, and $U^{\text {s }}$ denote the subgroups $\mathbf{p}^{-1}(P), \mathbf{p}^{-1}(M)$, and $\mathbf{s}(U)$ of $\widetilde{G}$, respectively. Then $\widetilde{P}=\widetilde{M} U^{\mathrm{s}}$, and $\widetilde{M} \cap U^{\mathbf{s}}=\{\tilde{e}\}$, where $\tilde{e}$ is the identity of $\widetilde{G}$. 
Let $W_{M}:=W \cap M$. As in $\S 1$ of [2], let:

$$
\left[W / W_{M}\right]:=\left\{w \in W \mid w \text { is of minimal length in } w W_{M} \in W / W_{M}\right\},
$$

and let $w_{M}$ denote the longest element of $\left[W / W_{M}\right]$. Let $N_{M}:=N \cap M$, and let $N_{M}^{\mathbf{s}}:=\mathbf{s}\left(N_{M}\right)$. Since $w_{M} N_{M} w_{M}^{-1} \subseteq N$, we can a define a character $\psi_{M}^{*}: N_{M}^{\mathrm{s}} \rightarrow \mathbb{C}^{\times}$by:

$$
\psi_{M}^{*}(n):=\psi\left(w_{M} \mathbf{p}(n) w_{M}^{-1}\right), \quad \forall n \in N_{M}^{\mathbf{s}} .
$$

Let $\bar{\psi}_{M}^{*}$ be the character obtained from $\psi_{M}^{*}$ by complex conjugation. In particular, we have that $N_{G}^{\mathbf{s}}=N^{\mathbf{s}}$, and $w_{G}=e$, hence $\psi_{G}^{*}=\psi^{*}$, and $\bar{\psi}_{G}^{*}=\bar{\psi}^{*}$.

Let $\Pi_{M}^{\psi}$ denote the full-induced representation $\operatorname{Ind}\left(N_{M}^{\mathbf{s}}, \widetilde{M} ; \psi_{M}^{*}\right)$ of $\widetilde{M}$. The space $\mathcal{W}_{M}^{\psi}$ of $\Pi_{M}^{\psi}$ consists of the locally-constant functions $f: \widetilde{M} \rightarrow \mathbb{C}$ that satisfy $f(n g)=\psi_{M}^{*}(n) f(g)$ for all $n \in N_{M}^{\text {s }}$ and $g \in \widetilde{M}$, and $\widetilde{M}$ acts on $\mathcal{W}_{M}^{\psi}$ by right translation. Similarly, let ${ }^{\circ} \Pi_{M}^{\psi}$ denote the compactly-induced representation $\operatorname{Ind}^{\circ}\left(N_{M}^{\mathrm{s}}, \widetilde{M} ; \bar{\psi}_{M}^{*}\right)$ of $\widetilde{M}$. The space ${ }^{\circ} \mathcal{W}_{M}^{\bar{\psi}}$ of ${ }^{\circ} \Pi_{M}^{\bar{\psi}}$ consists of the locally-constant functions $f: \widetilde{M} \rightarrow \mathbb{C}$ that are compactly supported modulo $N_{M}^{\mathbf{s}}$ and satisfy $f(n g)=\bar{\psi}_{M}^{*}(n) f(g)$ for all $n \in N_{M}^{\mathbf{s}}$ and $g \in \widetilde{M}$. Then $\widetilde{M}$ also acts on ${ }^{\circ} \mathcal{W}_{M}^{\bar{\psi}}$ by right translation. By Proposition 2.25 (c) of $[\mathbf{1}],{ }^{\circ} \Pi_{M}^{\bar{\psi}}$ is the contragredient of the representation $\Pi_{M}^{\psi}$.

Let $\pi_{M}$ be a smooth representation of $\widetilde{M}$. A subspace $\mathcal{W}$ of $\mathcal{W}_{M}^{\psi}$ is said to be a $\psi_{M}^{*}$-Whittaker model for $\pi_{M}$ if $\mathcal{W}$ is $\widetilde{M}$-invariant, and the restriction of $\Pi_{M}^{\psi}$ to $\mathcal{W}$ is a representation that is equivalent to $\pi_{M}$. In other words, $\mathcal{W}$ is the image of an injective element of $\operatorname{Hom}_{\widetilde{M}}\left(\pi_{M}, \Pi_{M}^{\psi}\right)$.

The following theorem is a generalization to the metaplectic group of Rodier's theorem on the heredity of Whittaker models (cf. Theorem 2 of $[4])$.

Theorem. Let $\pi_{M}$ be an admissible representation of $\widetilde{M}$. Extend $\pi_{M}$ to a representation $\pi_{P}$ of $\widetilde{P}$ by letting $U^{\mathrm{s}}$ act trivially, and let $\pi_{G}$ be the normalized induced representation $\operatorname{Ind}\left(\widetilde{P}, \widetilde{G} ; \pi_{P}\right)$ of $\widetilde{G}$. Then $\operatorname{Hom}_{\widetilde{G}}\left(\pi_{G}, \Pi_{G}^{\psi}\right) \cong$ $\operatorname{Hom}_{\widetilde{M}}\left(\pi_{M}, \Pi_{M}^{\psi}\right)$.

Proof. A topological space $X$ is an l-space if it is Hausdorff, locally-compact, and zero-dimensional (cf. $\S 1.1$ of [1]). For any $l$-space $X$ and any complex vector space $V$, let $\mathcal{S}(X ; V)$ denote the space of locally-constant, compactlysupported functions from $X$ to $V$, and let $\mathcal{D}(X ; V)$ be the linear dual of $\mathcal{S}(X ; V)$. When $V=\mathbb{C}$, we will simply write $\mathcal{S}(X)$ and $\mathcal{D}(X)$, respectively. Any element of $\mathcal{D}(X ; V)$ [resp. $\mathcal{D}(X)$ ] is called a $V$-distribution [resp. distribution]. 
Let $\mathcal{V}$ denote the space of $\pi_{M}$. For any $l$-subspace $X$ of $\widetilde{G}$ such that $N^{\mathrm{s}} X \widetilde{P}=X$, let $\mathcal{D}^{1}(X)$ denote the space of $\mathcal{V}$-distributions $D \in \mathcal{D}(X ; \mathcal{V})$ that satisfy:

$$
\begin{aligned}
D\left(\lambda_{N}^{1}(n) \rho_{P}^{1}(p) \varphi\right)=\bar{\psi}^{*}(n) \delta(p)^{-1 / 2} D\left(\pi_{P}\left(p^{-1}\right) \circ \varphi\right), & \\
& \forall n \in N^{\mathbf{s}}, p \in \widetilde{P}, \varphi \in \mathcal{S}(X ; \mathcal{V}) .
\end{aligned}
$$

Here $\lambda_{N}^{1}: N^{\mathbf{s}} \rightarrow \operatorname{Aut}(\mathcal{S}(X ; \mathcal{V}))$ and $\rho_{P}^{1}: \widetilde{P} \rightarrow \operatorname{Aut}(\mathcal{S}(X ; \mathcal{V}))$ are the representations defined in the usual way by left and right translation, respectively, and $\delta: \widetilde{P} \rightarrow \mathbb{C}^{\times}$is the modular character of $\widetilde{P}$.

By a theorem of F. Bruhat (cf. Theorem 4 of $[4]), \mathcal{D}^{1}(\widetilde{G})$ is isomorphic to the space $\operatorname{Bil}_{\widetilde{G}}\left({ }^{\circ} \Pi_{G}^{\bar{\psi}}, \pi_{G}\right)$ of $\widetilde{G}$-invariant bilinear forms on ${ }^{\circ} \mathcal{W}_{G}^{\bar{\psi}} \times \mathcal{V}$ (i.e., intertwining forms in the sense of $\S 1$ of [4]). Here we have used the fact that $\widetilde{G}=\widetilde{P} K$ for some compact, open subset $K$ of $\widetilde{G}$, and that $\bar{\psi}_{G}^{*}=\bar{\psi}^{*}$. Since ${ }^{\circ} \Pi_{G}^{\bar{\psi}}$ is the contragredient of the representation $\Pi_{G}^{\psi}$, it can also be shown that $\operatorname{Hom}_{\widetilde{G}}\left(\pi_{G}, \Pi_{G}^{\psi}\right) \cong \operatorname{Bil}_{\widetilde{G}}\left({ }^{\circ} \Pi_{G}^{\bar{\psi}}, \pi_{G}\right)$. Hence, $\mathcal{D}^{1}(\widetilde{G}) \cong \operatorname{Hom}_{\widetilde{G}}\left(\pi_{G}, \Pi_{G}^{\psi}\right)$, and it remains to show that $\mathcal{D}^{1}(\widetilde{G}) \cong \operatorname{Hom}_{\widetilde{M}}\left(\pi_{M}, \Pi_{M}^{\psi}\right)$.

For every $w \in\left[W / W_{M}\right]$, let $\tilde{w}:=\mathbf{s}(w)$. Starting from the Bruhat decomposition for $G$, one can show that $\widetilde{G}=\coprod_{w} N^{\mathbf{s}} \tilde{w} \widetilde{P}$, where the disjoint union is taken over all $w \in\left[W / W_{M}\right]$ (cf. $\S 1$ of $\left.[2]\right)$. In order to describe $\mathcal{D}^{1}(\widetilde{G})$, it will suffice to study each space $\mathcal{D}^{1}\left(N^{\mathbf{s}} \tilde{w} \widetilde{P}\right)$ separately. Thus, let $w$ be a fixed element of $\left[W / W_{M}\right]$. For every $\varphi \in \mathcal{S}\left(N^{\mathbf{s}} \times \widetilde{P} ; \mathcal{V}\right)$, let $\hat{\varphi} \in \mathcal{S}\left(N^{\mathbf{s}} \tilde{w} \widetilde{P} ; \mathcal{V}\right)$ be defined by:

$$
\hat{\varphi}(n \tilde{w} p):=\int_{N^{\mathbf{s}} \cap \tilde{w} \widetilde{P} \tilde{w}^{-1}} \varphi\left(n n_{\circ}, \tilde{w}^{-1} n_{\circ}^{-1} \tilde{w} p\right) d n_{\circ}, \quad \forall n \in N^{\mathbf{s}}, p \in \widetilde{P},
$$

where $d n_{\circ}$ is a Haar measure for $N^{\mathbf{s}} \cap \tilde{w} \widetilde{P} \tilde{w}^{-1}$. The map $\varphi \mapsto \hat{\varphi}$ is surjective, hence by duality it follows that $\mathcal{D}^{1}\left(N^{\mathbf{s}} \tilde{w} \widetilde{P}\right)$ is isomorphic to the space $\mathcal{D}^{2}$ of $\mathcal{V}$-distributions $D \in \mathcal{D}\left(N^{\mathbf{s}} \times \widetilde{P} ; \mathcal{V}\right)$ that satisfy:

$$
\begin{aligned}
D\left(\lambda_{N}^{2}(n) \rho_{P}^{2}(p) \varphi\right) & =\bar{\psi}^{*}(n) \delta(p)^{-1 / 2} D\left(\pi_{P}\left(p^{-1}\right) \circ \varphi\right), & & \forall n \in N^{\mathbf{s}}, p \in \widetilde{P}, \\
D\left(\rho_{N}^{2}\left(n_{\circ}\right) \varphi\right) & =D\left(\lambda_{P}^{2}\left(\tilde{w}^{-1} n_{\circ} \tilde{w}\right) \varphi\right), & & \forall n_{\circ} \in N^{\mathbf{s}} \cap \tilde{w} \widetilde{P} \tilde{w}^{-1},
\end{aligned}
$$

for all $\varphi \in \mathcal{S}\left(N^{\mathbf{s}} \times \widetilde{P} ; \mathcal{V}\right)$. Here $\lambda_{N}^{2}$ and $\lambda_{P}^{2}$ are representations defined by left translation:

$$
\lambda_{N}^{2}: N^{\mathbf{s}} \rightarrow \operatorname{Aut}\left(\mathcal{S}\left(N^{\mathbf{s}} \times \widetilde{P} ; \mathcal{V}\right)\right), \quad \lambda_{P}^{2}: \widetilde{P} \rightarrow \operatorname{Aut}\left(\mathcal{S}\left(N^{\mathbf{s}} \times \widetilde{P} ; \mathcal{V}\right)\right),
$$

and $\rho_{N}^{2}$ and $\rho_{P}^{2}$ are representations defined by right translation:

$$
\rho_{N}^{2}: N^{\mathbf{s}} \rightarrow \operatorname{Aut}\left(\mathcal{S}\left(N^{\mathbf{s}} \times \widetilde{P} ; \mathcal{V}\right)\right), \quad \rho_{P}^{2}: \widetilde{P} \rightarrow \operatorname{Aut}\left(\mathcal{S}\left(N^{\mathbf{s}} \times \widetilde{P} ; \mathcal{V}\right)\right) .
$$


Next, we identify $\mathcal{S}\left(N^{\mathbf{s}} \times \widetilde{P} ; \mathcal{V}\right)$ with $\mathcal{S}\left(N^{\mathbf{s}}\right) \otimes \mathcal{S}(\widetilde{P} ; \mathcal{V})$ in the usual way, that is, for every $\varphi_{N} \in \mathcal{S}\left(N^{\mathbf{s}}\right)$ and $\varphi_{P} \in \mathcal{S}(\widetilde{P} ; \mathcal{V})$, let $\varphi_{N} \otimes \varphi_{P} \in \mathcal{S}\left(N^{\mathbf{s}} \times \widetilde{P} ; \mathcal{V}\right)$ be defined by:

$$
\left(\varphi_{N} \otimes \varphi_{P}\right)(n, p):=\varphi_{N}(n) \varphi_{P}(p), \quad \forall n \in N^{\mathbf{s}}, p \in \widetilde{P} .
$$

Let $\lambda_{N}^{3}$ and $\lambda_{P}^{3}$ be the representations defined by left translation:

$$
\lambda_{N}^{3}: N^{\mathbf{s}} \rightarrow \operatorname{Aut}\left(\mathcal{S}\left(N^{\mathbf{s}}\right)\right), \quad \lambda_{P}^{3}: \widetilde{P} \rightarrow \operatorname{Aut}(\mathcal{S}(\widetilde{P} ; \mathcal{V})),
$$

and let $\rho_{N}^{3}$ and $\rho_{P}^{3}$ be the representations defined by right translation:

$$
\rho_{N}^{3}: N^{\mathbf{s}} \rightarrow \operatorname{Aut}\left(\mathcal{S}\left(N^{\mathbf{s}}\right)\right), \quad \rho_{P}^{3}: \widetilde{P} \rightarrow \operatorname{Aut}(\mathcal{S}(\widetilde{P} ; \mathcal{V}))
$$

For every $D \in \mathcal{D}^{2}$ and $\varphi_{P} \in \mathcal{S}(\widetilde{P} ; \mathcal{V})$, let $D_{\varphi_{P}} \in \mathcal{D}\left(N^{\mathbf{s}}\right)$ be the distribution defined by $D_{\varphi_{P}}\left(\varphi_{N}\right):=D\left(\varphi_{N} \otimes \varphi_{P}\right)$ for all $\varphi_{N} \in \mathcal{S}\left(N^{\mathbf{s}}\right)$. Then $D_{\varphi_{P}}\left(\lambda_{N}^{3}(n) \varphi_{N}\right)=$ $\bar{\psi}^{*}(n) D_{\varphi_{P}}\left(\varphi_{N}\right)$ for all $\varphi_{N} \in \mathcal{S}\left(N^{\mathbf{s}}\right)$ and $n \in N^{\mathbf{s}}$, since $\lambda_{N}^{2}(n)\left(\varphi_{N} \otimes \varphi_{P}\right)=$ $\lambda_{N}^{3}(n) \varphi_{N} \otimes \varphi_{P}$. By the uniqueness of left quasi-invariant distributions on $N^{\mathrm{s}}$ (cf. $\S 1.18$ of [1] - the proof for quasi-invariant distributions is similar), it follows that $D_{\varphi_{P}}$ is a constant multiple of the distribution $\bar{\psi}^{*} d n \in \mathcal{D}\left(N^{\mathrm{s}}\right)$ defined by:

$$
\varphi_{N} \mapsto \int_{N^{\mathbf{s}}} \varphi_{N}(n) \bar{\psi}^{*}(n) d n, \quad \forall \varphi_{N} \in \mathcal{S}\left(N^{\mathbf{s}}\right) .
$$

Hence, $D_{\varphi_{P}}\left(\rho_{N}^{3}\left(n_{\circ}\right) \varphi_{N}\right)=\psi^{*}\left(n_{\circ}\right) D_{\varphi_{P}}\left(\varphi_{N}\right)$ for all $\varphi_{N} \in \mathcal{S}\left(N^{\mathbf{s}}\right)$ and $n_{\circ} \in$ $N^{\mathbf{s}} \cap \tilde{w} \widetilde{P} \tilde{w}^{-1}$, and it follows that $\mathcal{D}^{2}$ is isomorphic to the space $\mathcal{D}^{3}$ of $\mathcal{V}$ distributions $D \in \mathcal{D}(\widetilde{P} ; \mathcal{V})$ that satisfy:

$$
D\left(\lambda_{P}^{3}\left(p_{\circ}\right) \rho_{P}^{3}(p) \varphi\right)=\psi^{*}\left(\tilde{w} p_{\circ} \tilde{w}^{-1}\right) \delta(p)^{-1 / 2} D\left(\pi_{P}\left(p^{-1}\right) \circ \varphi\right)
$$

for all $p_{\circ} \in \tilde{w}^{-1} N^{\mathbf{s}} \tilde{w} \cap \widetilde{P}, p \in \widetilde{P}$, and $\varphi \in \mathcal{S}(\widetilde{P} ; \mathcal{V})$.

Proceeding as above, we next identify $\mathcal{S}(\widetilde{P} ; \mathcal{V})$ with $\mathcal{S}(\widetilde{M} ; \mathcal{V}) \otimes \mathcal{S}\left(U^{\mathbf{s}}\right)$. Thus, for every $\varphi_{M} \in \mathcal{S}(\widetilde{M} ; \mathcal{V})$ and $\varphi_{U} \in \mathcal{S}\left(U^{\mathbf{s}}\right), \varphi_{M} \otimes \varphi_{U} \in \mathcal{S}(\widetilde{P} ; \mathcal{V})$ is defined by:

$$
\left(\varphi_{M} \otimes \varphi_{U}\right)(m u):=\varphi_{M}(m) \varphi_{U}(u), \quad \forall m \in \widetilde{M}, u \in U^{\mathrm{s}} .
$$

Let $\lambda_{M}^{4}$ and $\lambda_{U}^{4}$ be the representations defined by left translation:

$$
\lambda_{M}^{4}: \widetilde{M} \rightarrow \operatorname{Aut}(\mathcal{S}(\widetilde{M} ; \mathcal{V})), \quad \lambda_{U}^{4}: U^{\mathbf{s}} \rightarrow \operatorname{Aut}\left(\mathcal{S}\left(U^{\mathbf{s}}\right)\right),
$$

and let $\rho_{M}^{4}$ and $\rho_{U}^{4}$ be the representations defined by right translation:

$$
\rho_{M}^{4}: \widetilde{M} \rightarrow \operatorname{Aut}(\mathcal{S}(\widetilde{M} ; \mathcal{V})), \quad \rho_{U}^{4}: U^{\mathbf{s}} \rightarrow \operatorname{Aut}\left(\mathcal{S}\left(U^{\mathbf{s}}\right)\right)
$$


If $D \in \mathcal{D}^{3}$ and $\varphi_{M} \in \mathcal{S}(\widetilde{M} ; \mathcal{V})$, let $D_{\varphi_{M}} \in \mathcal{D}\left(U^{\mathbf{s}}\right)$ be defined by $D_{\varphi_{M}}\left(\varphi_{U}\right):=$ $D\left(\varphi_{M} \otimes \varphi_{U}\right)$ for all $\varphi_{U} \in \mathcal{S}\left(U^{\mathrm{s}}\right)$. Since $\left.\delta\right|_{U^{\mathrm{s}}}=1$ and $\left.\pi_{P}\right|_{U^{\mathrm{s}}}=1$ :

$$
\begin{aligned}
D_{\varphi_{M}}\left(\rho_{U}^{4}(u) \varphi_{U}\right) & =D\left(\varphi_{M} \otimes \rho_{U}^{4}(u) \varphi_{U}\right)=D\left(\rho_{P}^{3}(u)\left(\varphi_{M} \otimes \varphi_{U}\right)\right) \\
& =D\left(\varphi_{M} \otimes \varphi_{U}\right)=D_{\varphi_{M}}\left(\varphi_{U}\right)
\end{aligned}
$$

for all $\varphi_{U} \in \mathcal{S}\left(U^{\mathbf{s}}\right)$ and $u \in U^{\mathbf{s}}$. Then by the uniqueness of right-invariant distributions on $U^{\mathrm{s}}$ (cf. $\S 1.18$ of [1]), it follows that $D_{\varphi_{M}}$ is a constant multiple $D^{\prime}\left(\varphi_{M}\right)$ of the Haar measure $d u \in \mathcal{D}\left(U^{\mathbf{s}}\right)$ :

$$
D\left(\varphi_{M} \otimes \varphi_{U}\right)=D^{\prime}\left(\varphi_{M}\right) \int_{U^{\mathrm{s}}} \varphi_{U}(u) d u, \quad \forall \varphi_{M} \in \mathcal{S}(\widetilde{M} ; \mathcal{V}), \varphi_{U} \in \mathcal{S}\left(U^{\mathbf{s}}\right),
$$

and $D^{\prime}$ is a $\mathcal{V}$-distribution in $\mathcal{D}(\widetilde{M} ; \mathcal{V})$.

We will now show that if $w \neq w_{M}$, then $\mathcal{D}^{1}\left(N^{\mathbf{s}} \tilde{w} \widetilde{P}\right)=0$. Let $D \in \mathcal{D}^{3}$ be fixed, and let $D^{\prime} \in \mathcal{D}(\widetilde{M} ; \mathcal{V})$ be as above. For every $\varphi \in \mathcal{S}(\widetilde{P} ; \mathcal{V})$, let $\varphi^{\prime} \in \mathcal{S}(\widetilde{M} ; \mathcal{V})$ be defined by:

$$
\varphi^{\prime}(m):=\int_{U^{\mathrm{s}}} \varphi(m u) d u, \quad \forall m \in \widetilde{M} .
$$

Then $D^{\prime}\left(\varphi^{\prime}\right)=D(\varphi)$ for all $\varphi \in \mathcal{S}(\widetilde{P} ; \mathcal{V})$. Indeed, this is easy to check when $\varphi$ is of the form $\varphi_{M} \otimes \varphi_{U}$, and the general case follows by linearity. Since $\widetilde{M}$ normalizes $U^{\mathrm{s}}$, it follows that $\left(\lambda_{P}^{3}(u) \varphi\right)^{\prime}=\varphi^{\prime}$ for all $\varphi \in \mathcal{S}(\widetilde{P} ; \mathcal{V})$ and $u \in U^{\mathbf{s}}$. In particular:

$$
D(\varphi)=D^{\prime}\left(\varphi^{\prime}\right)=D^{\prime}\left(\left(\lambda_{P}^{3}\left(u_{\circ}\right) \varphi\right)^{\prime}\right)=D\left(\lambda_{P}^{3}\left(u_{\circ}\right) \varphi\right)=\psi^{*}\left(\tilde{w} u_{\circ} \tilde{w}^{-1}\right) D(\varphi)
$$

for all $u_{\circ} \in \tilde{w}^{-1} N^{\mathbf{s}} \tilde{w} \cap U^{\mathbf{s}}$. Since $w$ is not the longest element in $[W /$ $\left.W_{M}\right]$, there exists a positive simple root $\alpha$ such that the root group $N_{\alpha}$ is contained in $w U w^{-1}$. Since $\psi$ is principal, we have that $\left.\psi^{*}\right|_{N_{\alpha}^{s}} \neq 1$. Moreover, from the definition of the Matsumoto 2-cocycle $\sigma$, it follows that $\tilde{w}^{-1} N_{\alpha}^{\mathbf{s}} \tilde{w}=\mathbf{s}\left(w^{-1} N_{\alpha} w\right)$, and $\tilde{w}^{-1} N^{\mathbf{s}} \tilde{w} \cap U^{\mathbf{s}}=\mathbf{s}\left(w^{-1} N w \cap U\right)$. Hence there exists a $u_{\circ} \in \tilde{w}^{-1} N_{\alpha}^{\mathbf{s}} \tilde{w} \subseteq \tilde{w}^{-1} N^{\mathbf{s}} \tilde{w} \cap U^{\mathbf{s}}$ such that $\psi^{*}\left(\tilde{w} u_{\circ} \tilde{w}^{-1}\right) \neq 1$. This shows that $D=0$, and therefore $\mathcal{D}^{1}\left(N^{\mathbf{s}} \tilde{w} \widetilde{P}\right) \cong \mathcal{D}^{2} \cong \mathcal{D}^{3}=0$.

Now suppose that $w=w_{M}$. Then $\tilde{w}^{-1} N^{\mathbf{s}} \tilde{w} \cap \widetilde{P}=\tilde{w}^{-1} N^{\mathbf{s}} \tilde{w} \cap \widetilde{M}$, and $\mathcal{D}^{3}$ is isomorphic to the space $\mathcal{D}^{4}$ of $\mathcal{V}$-distributions $D \in \mathcal{D}(\widetilde{M} ; \mathcal{V})$ that satisfy:

$$
D\left(\lambda_{M}^{4}\left(m_{\circ}\right) \rho_{M}^{4}(m) \varphi_{M}\right)=\psi^{*}\left(\tilde{w} m_{\circ} \tilde{w}^{-1}\right) \delta(m)^{1 / 2} D\left(\pi_{M}\left(m^{-1}\right) \circ \varphi_{M}\right)
$$

for all $m_{\circ} \in \tilde{w}^{-1} N^{\mathbf{s}} \tilde{w} \cap \widetilde{M}, m \in \widetilde{M}$, and $\varphi_{M} \in \mathcal{S}(\widetilde{M} ; \mathcal{V})$. Here we have used the fact that:

$$
\rho_{P}^{3}(m)\left(\varphi_{M} \otimes \varphi_{U}\right)=\rho_{M}^{4}(m) \varphi_{M} \otimes \lambda_{U}^{4}\left(m^{-1}\right) \rho_{U}^{4}(m) \varphi_{U}
$$


for all $m \in \widetilde{M}, \varphi_{M} \in \mathcal{S}(\widetilde{M} ; \mathcal{V})$, and $\varphi_{U} \in \mathcal{S}\left(U^{\mathrm{s}}\right)$, which implies that:

$$
\left(\rho_{P}^{3}(m)\left(\varphi_{M} \otimes \varphi_{U}\right)\right)^{\prime}=\delta(m)\left(\rho_{M}^{4}(m) \varphi_{M} \otimes \varphi_{U}\right)^{\prime} .
$$

By another straightforward calculation with the 2-cocycle $\sigma, \tilde{w}^{-1} N^{\mathbf{s}} \tilde{w} \cap \widetilde{M}=$ $N_{M}^{\mathbf{s}}$. Thus, from the definition of $\bar{\psi}_{M}^{*}$, it follows that complex conjugation provides an isomorphism between $\mathcal{D}^{4}$ and the space $\mathcal{D}^{5}$ of $\mathcal{V}$-distributions $D \in \mathcal{D}(\widetilde{M} ; \mathcal{V})$ that satisfy:

$$
\begin{aligned}
D\left(\lambda_{M}^{4}\left(m_{\circ}\right) \rho_{M}^{4}(m) \varphi\right)=\bar{\psi}_{M}^{*}\left(n_{\circ}\right) \delta(m)^{-1 / 2} D\left(\left(\delta^{-1} \otimes \pi_{M}\right)\left(m^{-1}\right) \circ \varphi\right), & \\
& \forall n_{\circ} \in N_{M}^{\mathbf{s}}, m \in \widetilde{M},
\end{aligned}
$$

for all $\varphi \in \mathcal{S}(\widetilde{M} ; \mathcal{V})$. By Bruhat's theorem, $\mathcal{D}^{5} \cong \operatorname{Bil}_{\widetilde{M}}\left({ }^{\circ} \Pi_{M}^{\bar{\psi}}, \delta^{-1} \otimes \pi_{M}\right)$, the space of intertwining forms of ${ }^{\circ} \Pi_{M}^{\bar{\psi}}$ and $\delta^{-1} \otimes \pi_{M}$, which is isomorphic to $\operatorname{Hom}_{\widetilde{M}}\left(\delta^{-1} \otimes \pi_{M}, \Pi_{M}^{\psi}\right)$. Finally, since $\left.\delta\right|_{N_{M}^{\mathrm{s}}}=1$, the spaces $\operatorname{Hom}_{\widetilde{M}}\left(\delta^{-1} \otimes \pi_{M}\right.$, $\Pi_{M}^{\psi}$ ) and $\operatorname{Hom}_{\widetilde{M}}\left(\pi_{M}, \Pi_{M}^{\psi}\right)$ are isomorphic (although the representations $\delta^{-1} \otimes \pi_{M}$ and $\pi_{M}$ need not be). Thus, in the case $w=w_{M}$, we have that $\mathcal{D}^{1}\left(N^{\mathrm{s}} \tilde{w} \widetilde{P}\right) \cong \operatorname{Hom}_{\widetilde{M}}\left(\pi_{M}, \Pi_{M}^{\psi}\right)$.

As $\widetilde{G}$ is a finite covering of $G$, we have that $N^{\mathbf{s}} \tilde{w}_{M} \widetilde{P}$ is open in $\widetilde{G}$, since $N w_{M} P$ is open in $G$. Similarly, $X^{1}:=\coprod_{w \neq w_{M}} N^{\mathbf{s}} \tilde{w} \widetilde{P}$ is closed in $\widetilde{G}$, and the sequence:

$$
0 \rightarrow \mathcal{D}^{1}\left(X^{1}\right) \rightarrow \mathcal{D}^{1}(\widetilde{G}) \rightarrow \mathcal{D}^{1}\left(N^{\mathbf{s}} \tilde{w}_{M} \widetilde{P}\right) \rightarrow 0
$$

is exact (cf. $\S 1.9$ of $[1]$ ). We will now show that $\mathcal{D}^{1}\left(X^{1}\right)=0$. Indeed, for $i \geq 1$, let $X^{i+1}:=X^{i}-Y^{i}$, where:

$Y^{i}:=\coprod_{w \in W^{i}} N^{\mathbf{s}} \tilde{w} \widetilde{P}, \quad W^{i}:=\left\{w \in W / W_{M} \mid N^{\mathbf{s}} \tilde{w} \widetilde{P}\right.$ is an open subset of $\left.X^{i}\right\}$.

Then $W / W_{M}-\left\{w_{M}\right\}=\coprod_{i \geq 1} W^{i}$, and $X^{1}=\coprod_{i \geq 1} Y^{i}$. As each $Y^{i}$ is open in $X^{i}$, and each $X^{i+1}$ is closed in $X^{i}$, the sequence:

$$
0 \rightarrow \mathcal{D}^{1}\left(X^{i+1}\right) \rightarrow \mathcal{D}^{1}\left(X^{i}\right) \rightarrow \mathcal{D}^{1}\left(Y^{i}\right) \rightarrow 0
$$

is also exact. But $N^{\mathbf{s}} \tilde{w} \widetilde{P}$ is also an open subset of $Y^{i}$ for every $w \in W^{i}$, hence it follows that $\mathcal{D}^{1}\left(Y^{i}\right) \cong \bigoplus_{w \in W^{i}} \mathcal{D}^{1}\left(N^{\mathbf{s}} \tilde{w} \widetilde{P}\right)=0$. Consequently, $\mathcal{D}^{1}\left(X^{1}\right) \cong$ $\mathcal{D}^{1}\left(X^{i}\right)$ for all $i \geq 1$, and since $X^{i}=\emptyset$ for $i \gg 0$, this shows that $\mathcal{D}^{1}\left(X^{1}\right)=0$. From the exact sequence $(* *)$, it now follows that $\mathcal{D}^{1}(\widetilde{G}) \cong \mathcal{D}^{1}\left(N^{\mathrm{s}} \tilde{w}_{M} \widetilde{P}\right) \cong$ $\operatorname{Hom}_{\widetilde{M}}\left(\pi_{M}, \Pi_{M}^{\psi}\right)$, and this completes the proof. 


\section{References}

[1] I.N. Bernstein and A.V. Zelevinsky, Representations of the group $G L(n, F)$ where $F$ is a non-Archimedean local field, Russian Math. Surveys, 31(3) (1976), 1-68.

[2] W. Casselman, Introduction to the theory of admissible representations of $p$-adic reductive groups, Lecture Notes.

[3] D.A. Kazhdan and S.J. Patterson, Metaplectic forms, Publ. Math. IHES, 59 (1984), 35-142.

[4] F. Rodier, Whittaker models for admissible representations of reductive p-adic split groups, Proc. Symp. Pure Math., 26 (1973), 425-430.

[5] F. Shahidi, Local coefficients and normalization of intertwining operators for $G L(n)$, Comp. Math., 48(3) (1983), 271-295.

Received December 4, 1996 and revised April 28, 1997.

OKlahoma State University

STILLWATER, OK 74078-1058

E-mail address: wdb@littlewood.math.okstate.edu 